\title{
Three-Dimensional Micropatterned Cardiac Tissue Cultures
}

\author{
Andrew D. McCulloch,* John O. Gallagher,* Sangeeta N. Bhatia,* Thomas K. Borg, and Jeffrey H. \\ Omens*
}

* Departments of Bioengineering and Medicine, Whitaker Institute for Biomedical Engineering, University of California San Diego, 9500 Gilman Drive, La Jolla, CA 92093-0412

** Department of Cell and Developmental Biology and Anatomy, School of Medicine, University of South Carolina, Columbia, South Carolina 29208

A major challenge of tissue engineering is devise methods to culture tissue that has similar properties to the in vivo tissue. Tissue culture as opposed to cell culture, is usually composed of multiple cell types containing at least the parenchyma and connective tissue elements. In cardiac muscle, the predominant cell type is the fibroblast; however, the cardiac myocyte represents the largest volume of cellular mass. Design of specific substrates for the three-dimensional culture of cardiac myocytes has been a goal of our studies. Using microlithography and microfluidic patterning (Fig. 1), we are able to culture cardiac neonatal myocytes with an adult in vivo phenotype (Fig. 2). In addition, the cardiac fibroblasts also form an in vivo phenotype in this culture system. The proliferation of the fibroblast does not "over grow" the myocytes and forms a similar ratio as in vivo.

To investigate the effects of anisotropic physical strains on mechanotransduction in the in-vitro setting, cardiac myocytes have been cultured on topographically defined surfaces. Subsequent analysis of protein expression of bi-axially stretch myocytes has shown that levels key proteins involved in mechanotransduction are altered depending on which direction the myocytes are aligned when stretched. The expression levels of p38, Phosphorylated Erk 1/2 and Phosphorlyated FAK all appear to elicit different responses in relation to the direction of the principal strain. Downstream of these pathways, we see that an increased induction of connexin-43, N-cadherin and F-actin by stretch transverse to the myofilament axes compared with longitudinally (Fig. 3) [1].

To characterize the contractile function of these engineered cell cultures, a method to measure phasic contractile stresses in myocyte cell cultures adhered to substrate has been developed. Microfabrication techniques were used to pattern aligned myocytes in islands and we imaged the displacements of material markers in the underlying substrate and surrounding cell-free membrane to measure contractile stress development in myocytes. Neonatal myocytes were plated on soft elastomeric membranes thin enough that individual myocytes and clusters of myocytes could deform the substrate membrane. The displacements of fluorescent microspheres embedded in the elastomer were used to measure deformations.

Knowing the boundary conditions and elastomer material properties, these strains are used to compute regional cell forces in an extension of traction-force microscopy. Elastomeric sheets, $4 \mu \mathrm{m}$ thick, were coated with type-I collagen in patterns using microfluidic techniques and plated with one million primary rat neonatal ventricular myocytes in media containing serum. The displacements of $0.2 \mu \mathrm{m}$ diameter fluorescent microspheres embedded in the polydimethylsiloxane membrane were tracked with high-resolution microscopy at $30 \mathrm{fps}$. From the displacements of numerous markers in a cell-free region surrounding a myocyte, displacement fields were determined. 2-D strains are 
computed which showed phasic stretch and shortening of the substrate at the beating frequency of the myocytes.

\section{References}

[1] S.M. Gopalan et al., Biotechnol. Bioeng. 5 (2003) 578-87.

[2] This research was supported by the National Heart Lung and Blood Institute through NIH grant 5 R21 HL72160-03.

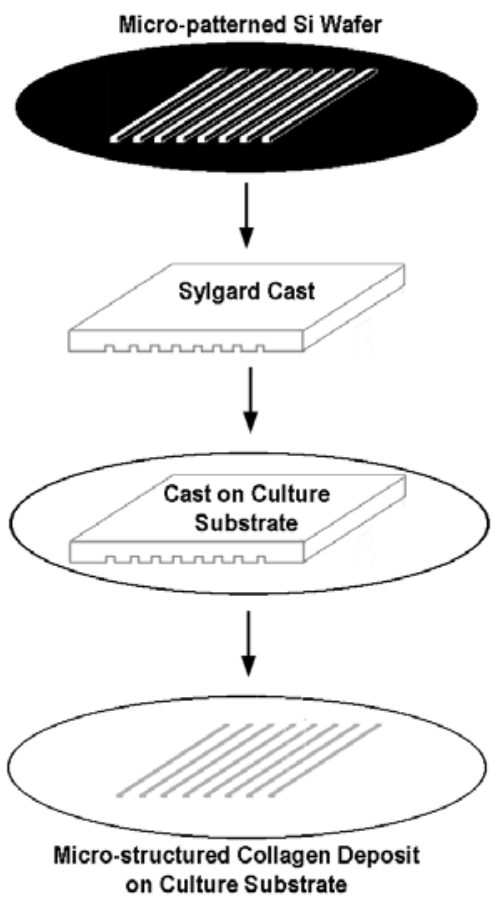

FIG. 1. Microlithography and microfluidic patterning of elastic substrata.

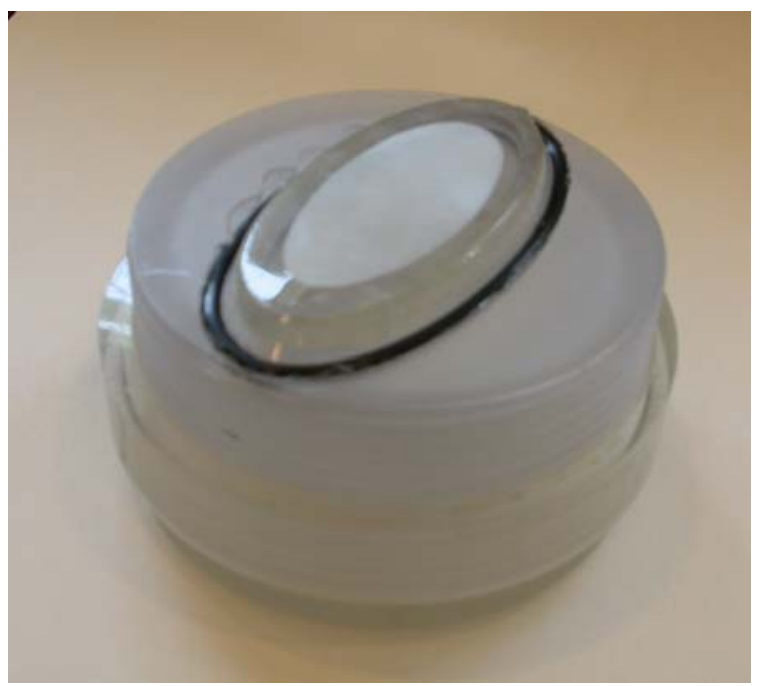

FIG. 3. Transverse stretch induces cell-cell coupling proteins more strongly than longitudinal stretch.

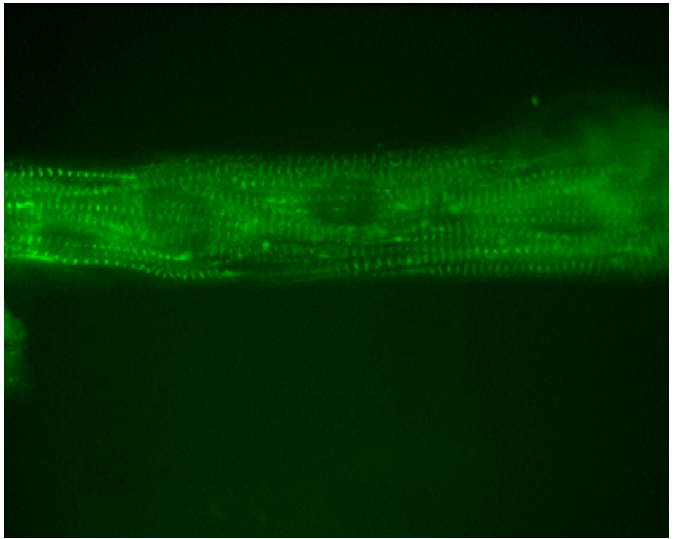

FIG. 2. Micropatterned neonatal myocyte.

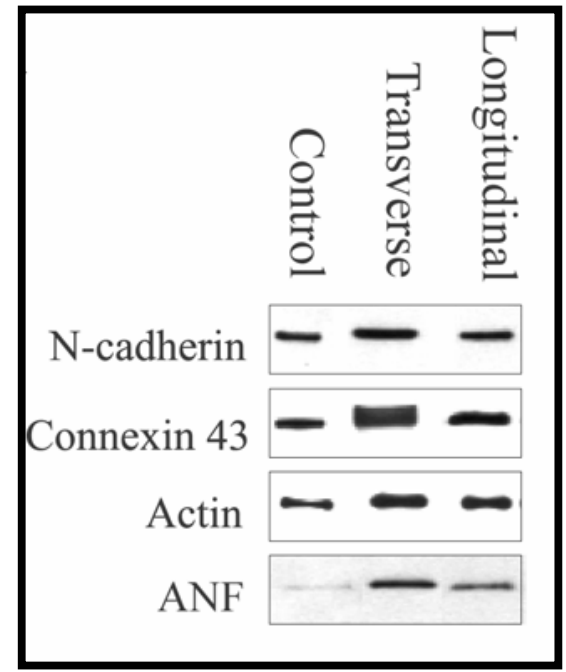

